\title{
SELLANTES DE FIBRINA EN GINECOLOGÍA LAPAROSCÓPICA. ASPECTOS MOLECULARES Y MECANISMO DE ACCIÓN: REVISIÓN DE LA LITERATURA
}

\section{Molecular aspects and mechanism of action regarding the use of fibrin sealants in laparoscopic gynecology: a literature review}

Hernando Navarro-Newball, M.D. *, William García-Gutiérrez**, Eliana Paredes-Becerra**

Recibido: mayo 4/11 - Aceptado: diciembre 19/11

\section{RESUMEN}

Objetivo: hacer una revisión sobre la actividad biológica y mecanismo de acción de los sellantes de fibrina en ginecología laparoscópica.

Materiales y métodos: se realizó una búsqueda de la literatura publicada tanto en inglés como en español a través de Medline vía PubMed, Ovid y Cochrane, desde 1993 a 2010. Se analizaron los artículos que incluían el uso de los sellantes en ginecología laparoscópica y se agruparon por temáticas tales como antecedentes, actividad biológica, composición, administración y características biofísicas.

Resultados: se encontraron entre 1993 y 2010, 200 artículos de los cuales 49 correspondían a los criterios establecidos siendo objeto de análisis. Los sellantes de fibrina contribuyen al logro de la hemostasia aumentando la expresión en las células peritoneales de los activadores e inhibidores del plasminógeno, afectando el proceso de curación,

* Ginecólogo Laparoscopista. Docente Universidad Libre, Cali (Colombia).Correo electrónico: hnavarro_newball@hotmail.com

** Residente de IV año Ginecología y Obstetricia Universidad Libre. Cali, (Colombia). que puede ser de beneficio en la reducción de las adherencias posoperatorias.

Conclusión: el desarrollo de los sellantes de fibrina surge como una alternativa práctica para la reducción de la hemorragia y control de la hemostasia, además de la reducción de las adherencias posoperatorias en la cirugía laparoscópica ginecológica.

Palabras clave: sellante de fibrina, hemostático, adhesivo, ginecología, laparoscopia.

\section{SUMMARY}

Objective: reviewing fibrin sealants' biological activity and mechanism of action related to laparoscopic gynecology.

Materials and methods: a search was made of the literature published in both English and Spanish via PubMed/Medline, OVID and Cochrane from 1993 to 2010. Articles involving the use of sealants in laparoscopic gynecology were analyzed and grouped by topic, such as background, biological activity, composition, administration and biophysical characteristics. 
Results: 200 articles were found which had been published from 1993 to 2010, 49 of them complied with the established analysis criteria. Fibrin sealants contributed towards achieving hemostasis, increasing plasminogen activator and inhibitor expression in peritoneal cells, thereby affecting curing, which could be beneficial in reducing post-operative adhesion.

Conclusion: developing fibrin sealants emerges as a practical alternative for reducing hemorrhage and controlling hemostasia, as well as reducing postoperative adhesion in surgery involving laparoscopic gynecology.

Key words: fibrin sealant, haemostatic, adhesive, gynecology, laparoscopy.

\section{INTRODUCCIÓN}

Con el auge de la laparoscopia se han incorporado nuevas técnicas y métodos de hemostasia dentro de los cuales los sellantes de fibrina ocupan un lugar muy importante por su facilidad de aplicación y resultados. ${ }^{1,2}$ Los sellantes de fibrina pertenecen al grupo de productos con propiedades adhesivas y hemostáticas derivados principalmente del plasma humano, contienen fibrinógeno, trombina, factor XIII, fibronectina, aprotinina y cloruro de calcio. ${ }^{3}$ Estos productos reproducen los pasos finales de la cascada de coagulación, formando un coágulo de fibrina estable, deteniendo la pérdida sanguínea y ayudando en el proceso normal de cicatrización. ${ }^{3}$

Con el propósito de brindar información al ginecólogo laparoscopista y a otros profesionales de la salud, que facilite el conocimiento y aplicación de estos productos se presenta esta revisión sobre la farmacología, el mecanismo acción y la actividad biológica de los sellantes de fibrina.

\section{MATERIALES Y MÉTODOS}

Con las palabras clave "sellantes de fibrina", "hemostasia", "ginecología” y "laparoscopia" se realizó una búsqueda en las bases de datos Medline vía PubMed, Ovid y Cochrane desde 1993 hasta 2010, en idiomas inglés y español.
Se analizaron los artículos relacionados con las características biofísicas y bioquímicas de los sellantes, administración, actividad biológica y del uso del dispositivo médico en laparoscopia ginecológica. Se clasificaron las revisiones bibliográficas de acuerdo con las variables mencionadas y se condensó la información en forma ordenada y secuencial.

\section{RESULTADOS}

Se encontraron 200 referencias de los cuales se analizaron 49 artículos porque se relacionaban con uso en humanos y laparoscopia ginecológica. Respecto al uso de sellantes en ginecología fueron incluidas 11 referencias.

\section{Características farmacológicas y mecanismo de acción}

El primer sellante de fibrina aprobado por la FDA en 1998 y el más estudiado en los Estados Unidos fue Tisseel o Tissucol ${ }^{\circledR}$ (Baxter), contiene aprotinina bovina que es un agente antifibrinolítico que frena la disolución natural del coágulo. ${ }^{1,4}$ Este sistema hemostático está constituido por dos componentes de origen humano: el concentrado proteico liofilizado, para disolver con solución de aprotinina, y la trombina liofilizada para reconstituir con solución de cloruro de calcio. Ambos componentes se elaboran a partir de una mezcla de plasma humano. ${ }^{5-7}$ El mecanismo de acción de los sellantes de fibrina corresponde a la última fase de la coagulación sanguínea. La molécula de fibrinógeno humana es una glicoproteína compuesta de tres pares de cadenas polipeptídicas $(\propto, \beta, \gamma)$ que forman una molécula con dos mitades simétricas. ${ }^{8}$ El fibrinógeno bajo la acción de la trombina se transforma en fibrina $(\propto, \beta, \gamma)$ con liberación de dos moléculas de fibrinopéptido A y B. Los monómeros de fibrina formados se polimerizan en dímeros y posteriormente se unen entre sí mediante enlaces covalentes, por acción del factor XIII previamente activado por la trombina y en presencia de iones de calcio. ${ }^{89}$ La fibrina producida se adhiere a los tejidos que resultan expuestos tras la lesión tisular, 
con especial afinidad por las fibras de colágeno. ${ }^{2}$ La malla de fibrina formada sirve de soporte para la proliferación de fibroblastos y capilares que se producen en el proceso de cicatrización. ${ }^{10}$ La última fase del proceso es la degradación por proteólisis y fagocitosis de la malla de fibrina, en la que intervienen los sellantes de fibrina. La fibrinólisis, entre otros factores, depende de la presencia de los activadores tisulares del plasminógeno, cuya concentración puede variar de un tejido a otro. Así, la etapa final es la sustitución de la malla de fibrina por tejido conjuntivo y la posterior formación de un tejido de cicatrización. ${ }^{6,7}$ Es en este punto en el que el tipo de sellante juega un papel relevante de acuerdo con la cantidad de fibrinógeno, plasminógeno y activador del plasminógeno tisular (t-PA). ${ }^{7}$

\section{Composición}

El liofilizado necesario para obtener $1 \mathrm{ml}$ de solución sellante de fibrina contiene los siguientes principios activos: proteína coagulable 75-115 mg; fibrinógeno 70-110 $\mathrm{mg}$; plasmafibronectina 2-9 mg; plasminógeno 40-120 mcg; factor XIII 10-50 UI; solución de aprotinina bovina we3.000 UIC/ml; trombina humana liofilizada; trombina 4: $1 \mathrm{ml}$ de la solución reconstituida contiene 4 UI; trombina 500: $1 \mathrm{ml}$ de la solución reconstituida contiene 500 UI; solución de cloruro de calcio $40 \mu \mathrm{mol} \mathrm{CaCI} / \mathrm{m}^{5,11-14}$

\section{Características biofísicas}

Las formas de fibrinógeno fotoquímicamente reticulado de los sellantes de tejidos son al menos 5 veces más fuertes (presión), (95,9 +/- 4,5 kilopascales $(\mathrm{kPa})$ o 19,501 g) que un sellador de fibrina convencional (17,9 +/-9,3 $\mathrm{kPa}$ o 3,640 g); con una fuerza tensil de $125 \mathrm{~g} / \mathrm{cm}^{2} \cdot{ }^{15-18}$ Cuando se comparan las características de varios agentes hemostáticos, se encuentra que las suturas son las que ofrecen mayor presión y pueden ser utilizadas en vasos de mayor calibre $(900 \mathrm{mmHg}$ y $7 \mathrm{~mm}$, respectivamente), seguidos por los clips de polímero (854 mmHg y $4 \mathrm{~mm}$ ), bipolar (601 mmHg y $4 \mathrm{~mm}$ ), endograpadora (310 mmHg y $17 \mathrm{~mm}$ ) y luego los sellantes de fibrina en parénquima $(378 \mathrm{mmHg})$, superiores al uso de monopolar (230 $\mathrm{mmHg}$ ) bisturí armónico (204 mmHg y 4 mm). ${ }^{15,19-25}$

\section{Actividad biológica}

Para evaluar la actividad del sellador de fibrina se realizó un ensayo clínico en el laboratorio de la Universidad de Detroit (Michigan) división de Ginecología y Obstetricia en el 2004, en donde se midió la expresión del ácido ribonucleico mensajero (ARNm) de factores reguladores de la coagulación como el activador del plasminógeno en las células peritoneales. ${ }^{8}$ La actividad del plasminógeno y del activador del plasminógeno tisular t-PA, se cree que juegan un papel fundamental en la degradación de la malla de fibrina que se desarrolla después de procedimientos quirúrgicos. La reducción de estos compuestos, como ocurre con el trauma de los tejidos, se traduce en un mayor desarrollo de adherencias posoperatorias. ${ }^{8}$ Se utilizó transcriptasa reversa (RT) y cambios en la reacción de polimerasa (PCR) para determinar los cambios de t-PA y del inhibidor del activador del plasminógeno 1 (PAI-1, por sus siglas en inglés) en seis condiciones, a las 6 , 12, 24 y 48 horas. El sellante de fibrina usado fue Tissucol, un sellante de fibrina en dos componentes en dilución 1 a 2, y cultivo control. No hubo cambios en los niveles de ARNm de t-PAy PAI-1 a las 48 horas. Se observó un aumento selectivo en los fibroblastos peritoneales normales; aumentos similares fueron identificados en los cultivos de fibroblastos. Los autores concluyen que la aprotinina utilizada como antifibrinolítico no aumenta la expresión en las células peritoneales tanto de la t-PA como del PAI-1, los cambios no se ven en concentraciones fisiológicas del sellante de fibrina. Estas observaciones sugieren que, además de su capacidad para contribuir al logro de la hemostasia, el sellante de fibrina afecta el proceso de curación mediante la alteración de los componentes del sistema activador del plasminógeno, que puede ser de beneficio en la reducción de las adherencias posoperatorias. ${ }^{8}$ 
En otro estudio realizado por la Universidad de Sao Paulo (Brasil), y publicado en el 2009, al realizar histerectomía abdominal en conejas, utilizaron sutura de ácido poliglicólico y sellantes de fibrina, se encontró mejor adherencia a la cúpula, con mejor tejido de granulación con el ácido poliglicólico, pero una mejor malla de fibrina y mayor presión de ruptura con los sellantes de fibrina, con lo que concluyen que el cierre de la cúpula vaginal utilizando la cola de fibrina es un procedimiento seguro y fiable después de la histerectomía abdominal en el modelo de conejas. ${ }^{11}$

\section{Administración}

Deben administrarse únicamente por vía tópica. La dosis requerida depende del tamaño de la superficie que debe cubrirse, con la presentación de $1 \mathrm{ml}$ - $2 \mathrm{ml}$ de solución, se cubre un área de unos $10 \mathrm{~cm}^{2}$ si se utiliza en cánula, y de $25 \mathrm{~cm}^{2}$ a $100 \mathrm{~cm}^{2}$, utilizando un equipo pulverizador. . $^{514-16,26}$ El sellante de fibrina se adhiere firmemente al tejido circundante entre 3 a 5 minutos, a los 10 minutos adquiere el 70\% de resistencia, la cual es máxima a las dos horas. ${ }^{5,17}$

\section{Usos en ginecología laparoscópica}

El uso de sellantes de fibrina durante la miomectomía laparoscópica podría reducir considerablemente el riesgo de adherencias. ${ }^{26,27}$ Los sellantes han aumentado su popularidad en las intervenciones para mejorar la hemostasia perioperatoria. Existen revisiones sistemáticas de ensayos controlados en los que pacientes adultos llevados a cirugía electiva se asignan al azar a manejo con sellantes o grupo sin tratamiento, en los que se encuentra que en el abordaje laparoscópico durante la miomectomía se disminuye un 36\% el riesgo de adherencias, en comparación con la laparotomía. ${ }^{13,15,26,28-30}$ Cuando se adiciona sellante de fibrina se disminuye el riesgo de adherencias de 6,5\% a 3\%, observado en la laparoscopia de control; además, hay una disminución del riesgo de hemorragia perioperatoria $(340 \mathrm{ml}$ frente a $110 \mathrm{ml}) \cdot{ }^{17,31,32}$

\section{CONCLUSIÓN}

Los sellantes de fibrina contribuyen al logro de la hemostasia aumentando tanto la expresión en las células del t-PA como del PAI-1, afectan el proceso de curación mediante la alteración de los componentes del sistema activador del plasminógeno, que puede ser de beneficio en la reducción de las adherencias posoperatorias.

\section{REFERENCIAS}

1. Busuttil RW. A comparison of antifibrinolytic agents used in hemostatic fibrin sealants. J Am Coll Surg 2003;197:1021-8.

2. Reece TB, Maxey TS, Kron IL. A prospectus on tissue adhesives. Am J Surg 2001;187:40S-44S.

3. Jackson MR, MacPhee MJ, Drohan WN, Alving BM. Fibrin sealant: current and potential clinical applications. Blood Coagul Fibrinol 1996;7:737-46.

4. Hackethal A, Brüggmann. Surgical treatment of fibroids in Hessel, Germany, between 1998 and 2004. Fertil Steril 2008;91:862-8.

5. Carr ME Jr, Martin EJ, Ambrose H. Fibrin sealants. En: Wnek GE, Bowlin GL, editors. Encyclopedia of Biomaterials and Biomedical Engineering. New York: Marcel Dekker; 2004. p. 611-20.

6. Diamond M, Kruger M, Saed GM. Effect of Tisseel on expression of tissue plasminogen activator and plasminogen activator inhibitor-1. Fertil Steril 2004;80:1657-64.

7. Dodd RA, Cornwell R, Holm NE, Garbarsch A, Hollingsbee DA. The Vivostat application system: a comparison with conventional fibrin sealant application systems. Technol Health Care 2002;10:401-11.

8. Bar L, Malka O, Naboichenko E, Nur I. The binding of fibrin sealant to collagen is influenced by the method of purification and the cross-linked fibrinogen-fibronectin (heteronectin) content of the 'fibrinogen' component. Blood Coagul Fibrinolysis 2005;16:111-7.

9. Rivoire HC, Fagundes DJ. Surgical adhesives and tubal sterilization: an experimental study. J Obstet Gynaecol Res 2008;34:218-27.

10. Petter-Puchner AH, Walder N, Redl H, Schwab R, Ohlinger W, Gruber-Blum S, et al. Fibrin sealant (Tissucol) enhances tissue integration of condensed polytetrafluoroethylene meshes and reduces early 
adhesion formation in experimental intraabdominal peritoneal onlay mesh repair. J Surg Res 2008;150:190-5.

11. Lovisetto F, Zonta S, Rota E, Mazzilli M, Bardone M, Bottero L, et al. Use of human fibrin glue (Tissucol) versus staples for mesh fixation in laparoscopic transabdominal preperitoneal hernioplasty: a prospective, randomized study. Ann Surg 2007;245:222-31.

12. Buchta C, Hedrich HC, Macher M, Höcker P, Redl H. Biochemical characterization of autologous fibrin sealants produced by CryoSeal and Vivostat in comparison to the homologous fibrin sealant product Tissucol/Tisseel. Biomaterials 2005;26:6233-41.

13. MacGillivray TE. Fibrin sealants and glues. J Card Surg 2003;18:480-5.

14. Lima AG, Taha M, Rivoire HC, Fagundes AT, Fagundes DJ. Fibrin adhesive and the vaginal vault synthesis on female rabbits abdominal hysterectomies. Acta Cir Bras 2009;24:30-5.

15. Sierra DH. Fibrin sealant adhesive systems: a review of their chemistry, material properties and clinical applications. J Biomater Appl 1993;7:309-52.

16. Elvin CM, Brownlee AG, Huson MG, Tebb TA, Kim M, Lyons RE, et al. The development of photochemically crosslinked native fibrinogen as a rapidly formed and mechanically strong surgical tissue sealant. Biomaterials 2009;30:2059-65.

17. Krishnan LK, Mohanty M, Umashankar PR, Lal AV. Comparative evaluation of absorbable hemostats: advantages of fibrin-based sheets. Biomaterials 2004;25:5557-63.

18. Weaver FA, Hood DB, Zatina M, Messina L, Badduke B. Gelatin-thrombin-based hemostatic sealant for intraoperative bleeding in vascular surgery. Ann Vasc Surg 2002;16:286-93.

19. Lee MG, Jones D. Applications of fibrin sealant in surgery. Surg Innov 2005;12:203-13.

20. Harrell AG, Kercher KW, Heniford BT. Energy sources in laparoscopy. Semin Laparosc Surg 2004;11:201-9.

21. McGinnis DE, Strup SE, Gomella LG. Management of hemorrhage during laparoscopy. J Endourol 2000;14:915-20.
22. Velada JL, Hollingsbee DA, Menzies AR, Cornwell R, Dodd RA. Reproducibility of the mechanical properties of Vivostat system patient-derived fibrin sealant. Biomaterials 2002;23:2249-54.

23. Canonico $\mathrm{S}$. The use of human fibrin glue in the surgical operations. Acta Biomed 2003;74:21-5.

24. Klingler CH, Remzi M, Marberger M, Janetschek G. Haemostasis in Laparoscopy Eur Urol 2006;50:948-57.

25. Alborzi S, Ghannadan E, Alborzi S, Alborzi M. A comparison of combined laparoscopic uterine artery ligation and myomectomy versus laparoscopic myomectomy in treatment of symptomatic myoma. Fertil Steril 2009;92:742-7.

26. Agdi M, Tulandi T. Endoscopic management of uterine fibroids. Best Pract Res Clin Obstet Gynaecol 2008;22:707-16.

27. Kongnyuy EJ, van den Broek N, Wiysonge CS. A systematic review of randomized controlled trials to reduce hemorrhage during myomectomy for uterine fibroids. Int J Gynaecol Obstet 2008;100:4-9.

28. Carless PA, Henry DA, Anthony DM. Uso de selladores de fibrina para reducir al mínimo la necesidad de transfusión perioperatoria de sangre alogénica, En: La Biblioteca Cochrane Plus, 2008 Número 4. Oxford: Update Software Ltd. Visitado 2011 Abr 25. Disponible en: http://www.updatesoftware.com

29. Takeuchi H, Kinoshita K. Evaluation of adhesion formation after laparoscopic myomectomy by systematic second-look microlaparoscopy. J Am Assoc Gynecol Laparosc 2002;9:442-6.

30. Kongnyuy EJ, Wilysonge CS. Interventions to reduce haemorrhage during myomectomy for fibroids. Cochrane Database Syst Rev 2007;24:CD005355.

31. Raga F, Sanz-Cortes M, Bonilla F, Casañ EM, BonillaMusoles F. Reducing blood loss at myomectomy with use of a gelatin-thrombin matrix hemostatic sealant. Fertil Steril 2009;92:356-60.

32. Saavedra J. Miomatosis uterina e infertilidad: indicaciones de tratamiento convencional. Rev Colomb Obstet Ginecol 2003:54;121-34. 